\title{
Comparative diagnostic imaging of a partial patellar ligament tear in a dog
}

\author{
Mario Ricciardi ${ }^{1, *}$ and Diana Lenoci ${ }^{2}$ \\ 1 "Pingry" Veterinary Hospital, via Medaglie d'Oro 5, Bari, Italy \\ ${ }^{2}$ Private Practitioner, Bari, Italy
}

\begin{abstract}
Traumatic lesions of the patellar ligament (PL) are rare in dogs. The resulting injury can be a complete or partial laceration, depending on the quantity of torn collagen fibres. Information obtained from imaging evaluation is of great value to the clinical approach towards PL injuries, because subsequent treatment options are affected by the distinction between complete or partial tears. Imaging diagnosis of PL damage in veterinary practice commonly relies on radiographic examination through the recognition of indirect signs, such as "patella alta", bone fragments at the level of the patellar or tibial insertion, and soft tissue opacity at the cranial aspect of the joint. Although ultrasound (US) and magnetic resonance imaging (MRI) have been described as useful diagnostic tools for the assessment of PL tears in human patients, specific comparative data regarding the evaluation of PL rupture in dogs using different imaging modalities is lacking in the veterinary literature. This paper describes the radiographic, ultrasonographic, CT and MRI imaging findings of a partial PL tear in a dog and discusses the utility of these techniques in diagnosing this condition. CT provided more detailed information than X-ray examination in the assessment of the osteoligamentous junction, the exclusion of microfracture and distal PL avulsion, but did not add information regarding PL integrity. MRI and US provided the most useful information regarding intra-ligamentous damage and as such their combined use may be considered for the assessment of PL injuries after clinical examination and survey radiographs.
\end{abstract}

Keywords: Computed tomography, Dog, Magnetic resonance imaging, Patellar ligament, Ultrasound.

\section{Introduction}

The patellar ligament (PL) is a robust fibrous extension of the quadriceps femoris muscle tendon, which connects the cranial and ventral patellar surface to the tibial tuberosity (Barone, 2003). This structure can be subjected to different types of lesion, most commonly of traumatic and inflammatory origin (Greis et al., 2005; Melloni et al., 2012), which are rarely reported in both veterinary and human orthopaedic practice (Gemmill and Carmichael, 2003; Das et al., 2014). Descriptions of PL injuries in dogs are limited to complete and partial ligament rupture (Gemmill and Carmichael, 2003; Das et al., 2014), of which direct trauma has been identified as by far the most common cause (Bloomberg and Parker, 1984; Culvenor, 1988; Aron et al., 1997). If traumatic force is placed directly on the PL, it may result in complete or partial laceration of the ligament fibrillary structure, depending on the quantity of torn collagen fibres. In such cases, imaging examinations may be particularly beneficial for injury confirmation, differentiation between complete and incomplete tears, for assessment of the degree of ligament disruption and hence the orientation of subsequent treatment strategies.

In veterinary practice, the imaging diagnosis of PL damage is based on radiographic examination, which is more commonly adopted than ultrasonography (Das et al., 2014). However, presumptive radiographic diagnosis of complete or partial PL rupture may only be reached through the recognition of indirect signs such as "patella alta", bone fragments at the patellar or tibial insertion level, as well as soft tissue opacity at the cranial joint aspect.

Radiography is generally considered less useful than ultrasound in assessing PL integrity, since it does not allow direct visualisation of the point of discontinuity in the PL structure (Das et al., 2014). On the other hand, the resolution of ultrasound images has improved rapidly in recent years. Ultrasound machines with highfrequency $(12-15 \mathrm{MHz})$ linear array transducers provide better image resolution and are widely used to evaluate superficial soft tissue structures such as muscle, tendon, ligament and bursae (Chiang et al., 2013).

However, in canine patients, ultrasonographic assessment of PL tears is limited to a small number of reported cases, with a detailed description of sonographic imaging findings lacking in the veterinary literature (Das et al., 2014).

In human medicine, magnetic resonance imaging (MRI), although endowed with inferior spatial resolution for tendineous structures compared to that of ultrasound, is considered the gold standard for tendon imaging, as it provides a complete anatomic overview 
of the articular region and excellent soft tissue contrast (Torriani and Kattapuram, 2003; Chang and Miller, 2009).

On the contrary, computed tomography (CT) does not offer any particularly great benefits in diagnostic terms for PL rupture characterisation (Schenck, 2001). Ultrasound, CT and MRI have been compared in terms of the evaluation of normal stifle anatomy in dogs, with similar advantages described for all techniques in the assessment of normal ligaments and tendons (Soler et al., 2007). However, specific comparative data regarding the evaluation of PL rupture using different imaging modalities are lacking in the small animal veterinary literature.

The aim of this paper was to describe and compare radiographic, ultrasonographic, CT and MRI imaging findings of a partial PL tear in a dog and to discuss the utility of such techniques in the diagnostic work-up of this condition.

\section{Case Details}

A 2-year-old intact male Vizsla, with normal activity level, was evaluated for a 1-month history of right hindlimb lameness following trauma of the stifle caused by forceful direct impact against a solid surface, which occurred during physical activity. Prior to the injury there had been no history of medical problems. Clinical examination findings included a reluctance to walk, grade 3 lameness based on the Sumner-Smith scale (Sumner-Smith, 1993), decreased weight-bearing on the right hind limb, swelling of the cranial aspect of the stifle, as well as pain on deep palpation and passive manipulation of the joint. Complete blood count, serum chemistry and urinalysis results were within normal limits.

After 5 days of antiflammatory therapy with robenacoxib $(2 \mathrm{mg} / \mathrm{Kg})$, a slight improvement in lameness and a reduction in tissue swelling were observed.

Orthogonal radiographic views of the right stifle joint were obtained under general anaesthesia. Medio-lateral views demonstrated soft tissue radiodensity at the cranial aspect of the joint at the level of the distal half of the PL. On medio-lateral view, only the proximal part of the PL was clearly seen with distinct margins, while its distal part, along with the cranio-ventral margin of the infrapatellar fat pad, was ill-defined and indistinguishable from the background of periligamentous soft tissue opacity (Fig. 1-A). Abnormalities in intra-ligamentous radiodensity were not seen. Bony abnormalities were not visible at the level of the tibial tuberosity. The cranio-caudal view was not useful for radiographic evaluation due to superimpositions.

In order to characterise the soft tissue joint damage and to assess PL integrity, after the radiographic examination an MRI was performed under general anaesthesia using a 0.25 Tesla permanent magnet (ESAOTE VET-MR GRANDE: Esaote, Genoa, Italy). The MRI sequence protocol included sagittal, transverse and dorsal Gradient Echo-STIR, sagittal T2weighted Fast Spin Echo, sagittal balanced steady-state free precession (bSSFP) sequence, and transverse and sagittal T1-weighted Spin echo, acquired before and after the intravenous administration of paramagnetic contrast medium (Magnegita ${ }^{\circledR}$ - gadopentate dimeglumine $500 \mathrm{mmol} / \mathrm{ml}$ - insight agents $\AA ; 0.15$ $\mathrm{mmol} / \mathrm{kg}$ BW: Agfa Healthcare Imaging Agents GmbH, Köln, Germany).

Magnetic resonance imaging revealed swelling of the distal half of the PL, with increased intra-ligamentous signal intensity in fat-suppressed sequences. The damaged part of the PL was surrounded by irregularly marginated pathologic material, isointense on the T1- and T2-weighted sequences with inhomogeneous contrast-enhancement, resembling a clot (Fig. 1-C,D). The abnormal material was seen more evidently adjacent to the inner surface of the PL, displacing the cranial aspect of the infrapatellar fat pad. Transverse spin-echo and bSSFP sequences oriented at the level of the distal PL insertion showed a focal linear defect in the medial area of the ligament section, originating from its caudal margin and extending toward the cranial margin. The cranial extension of the tear and involvement of the cranial ligament margin were not clearly assessable.

Multiplanar reformatting of the volumetric bSSFP sequence aligned according to the inner mid-dorsal axis of the PL showed complete disto-proximal extension of the lesion from the osteotendineous junction on the tibial tuberosity to the middle third of the PL (Fig. 2). At the level of the PL insertion, the tibial tuberosity showed a focal area of intraosseous pathological hyperintense signal in fat-suppressed sequences, compatible with a bone-marrow edema-like lesion (Starr et al., 2008) (Fig. 1-D).

In order to clarify any possible radiographically undetectable bone damage to the osteoligamentous junction at the level of the tibial tuberosity, an unenhanced CT scan of the right stifle was acquired immediately after the MRI examination, using a 16 slice-MDCT scanner (Somatom Emotion: Siemens, Forchheim, Germany) with the patient in sternal recumbency on the $\mathrm{CT}$ table under general anaesthesia. CT technical parameters were as follows: bone and standard acquisition algorithm, $110 \mathrm{kVp}, 180 \mathrm{mAs}$, 0.6$\mathrm{mm}$ slice thickness, pitch of 0.8 and $1 \mathrm{~s} /$ rotation. Threedimensional (3D) multiplanar reformatted and volumerendered images were obtained using dedicated 3D software (OsiriX DICOM-viewer: Pixmeo, Geneva, Switzerland). CT scans showed increased attenuation of the tibial tuberosity cancellous bone, with no sign of bone fracture or detached bone fragments at the 
osteoligamentous junction. The distal PL half was enlarged and surrounded by periligamentous accumulation of irregularly marginated material isoattenuating to the soft tissue. Such periligamentous swelling impeded identification of the boundaries of the distal PL half and exerted a compressive effect on the infrapatellar fat pad, which was caudally displaced. Discontinuities in PL structure were not evident (Fig. 1-B; Fig. 2-B,D; Fig. 3-F,G).

For a more detailed characterisation of the ligamentous injury the dog was subjected to ultrasonography of the stifle. B-mode ultrasound examination was performed with the dog maintained in lateral recumbency, using a portable ultrasound machine (MyLab TM Alpha, Esaote, Genoa, Italy) and a linear, 10-15 MHz high frequency transducer. Keeping the stifle normally flexed, the PL was examined from its cranial origin to its distal insertion in both the long and short axis planes. Transverse images were obtained with the ultrasound beam placed perpendicular to PL fibres, and longitudinal ultrasound images obtained by keeping the probe edge positioned over the patella. The scan technique was also extended over the lateral and medial sides of the PL. For dynamic ligament assessment, passive movements of flexion and extension were performed on the stifle joint while the ultrasound probe was held to the PL at different levels. Ultrasound videotapes and static images were collected and reviewed by both authors consensually.

The distal PL area appeared thickened with heterogeneous echotexture and a loss of fibrillary pattern (Fig. 1-E,F). At this site a $10 \mathrm{~mm}$ (proximodistal) anechoic defect, extending from the distal insertion to the midportion of the ligament, was detected within the PL fibres. The fibrillary defect appeared, on transverse images obtained at the level of the middle portion of the PL, as a $2 \mathrm{~mm}$ wide hypoechoic line in the medial half of the sections running from the caudal ligament margin to its centre. At the distal insertion level the defect measured $3.8 \mathrm{~mm}$ and affected the entire PL section from caudal to cranial margin (Fig. 3-I,L). The paratenon was not affected by the lesion.

Behind the patellar tendon the infrapatellar fat pad appeared inhomogeneously hyperechoic and caudally displaced by an accumulation of anechoic fluid interposed between the distal part of the PL and the tibial epiphysis (Fig. 1-E,F).

Outcome

The patient was discharged with an orthopaedic rigid knee brace. 30-day suspension of physical activity was recommended. One month later the dog appeared in good physical condition with significant clinical improvement of the hind limb lameness. Follow-up ultrasonography was recommended to monitor PL healing.

\section{Discussion}

A healthy PL is composed of parallel, longitudinally oriented type I collagen fibres $(86 \%)$ closely packed together and embedded in an amorphous matrix of proteoglycans $(1-5 \%)$ and inorganic components (0.2\%) (Lin et al., 2004). The PL does not have a tendon sheath but is instead surrounded by loose areolar connective tissue known as the paratenon (Chang and Miller, 2009; McNally, 2014). This histological structure makes the PL more similar to a tendon than a ligament (ligaments have more rounded cells and more type III collagen fibres than tendons) (Hall, 2007). Due to its histologic composition, the imaging appearance of the PL notably varies depending on the imaging technique adopted (radiographs, CT, MRI or US), with subsequent different grades of achievable detail. Diagnostic information obtained from imaging evaluation of the PL is of great value to the clinical approach towards traumatic injuries. In cases of stifle trauma with extensor mechanism injuries and presumptive PL damage, clinical investigation aims to: 1) confirm the presence of a ligament tear and 2) distinguish between partial and complete tears. Such a distinction affects subsequent treatment options; as described in the human medical literature, whereas incomplete ruptures with intact function may be treated nonoperatively with full extension immobilisation, complete ruptures require surgical treatment (Rosso et al., 2015).

Plain radiographs have low specificity for diagnosing PL injuries, whose recognition is thus based essentially on the detection of indirect signs (Das et al., 2014). In a study of 43 cases of PL injury in dogs, patella alta was considered the most reliable radiographic finding suggestive of PL rupture. However, in 2 patients in this group, the patella appeared normally positioned (Das et al., 2014).

The most effective method with which to diagnose patella alta in dogs appears to be the Insall-Salvati index, which is obtained by dividing the greatest diagonal length of the patella by the length of the PL. Normally this ratio is equal to 1.0 , with values $<0.80$ consistent with patella alta (Insall and Salvati, 1971). Disadvantages associated with this method include difficulty in assessing PL insertion on routine lateral radiographs, as well as the fact that its sensitivity may be influenced by variation in patellar morphology (Mostafa, 2008).

In our case, the distal half of the PL and its tibial insertion were ill-defined and indistinguishable from the background of the periligamentous soft tissue opacity, impeding the calculation of the Insall-Salvati ratio. Furthermore, as also noticed in the present study, the detection of true PL discontinuity on $\mathrm{x}$-ray examination is typically considered unreliable or inconsistent (Das et al., 2014). 

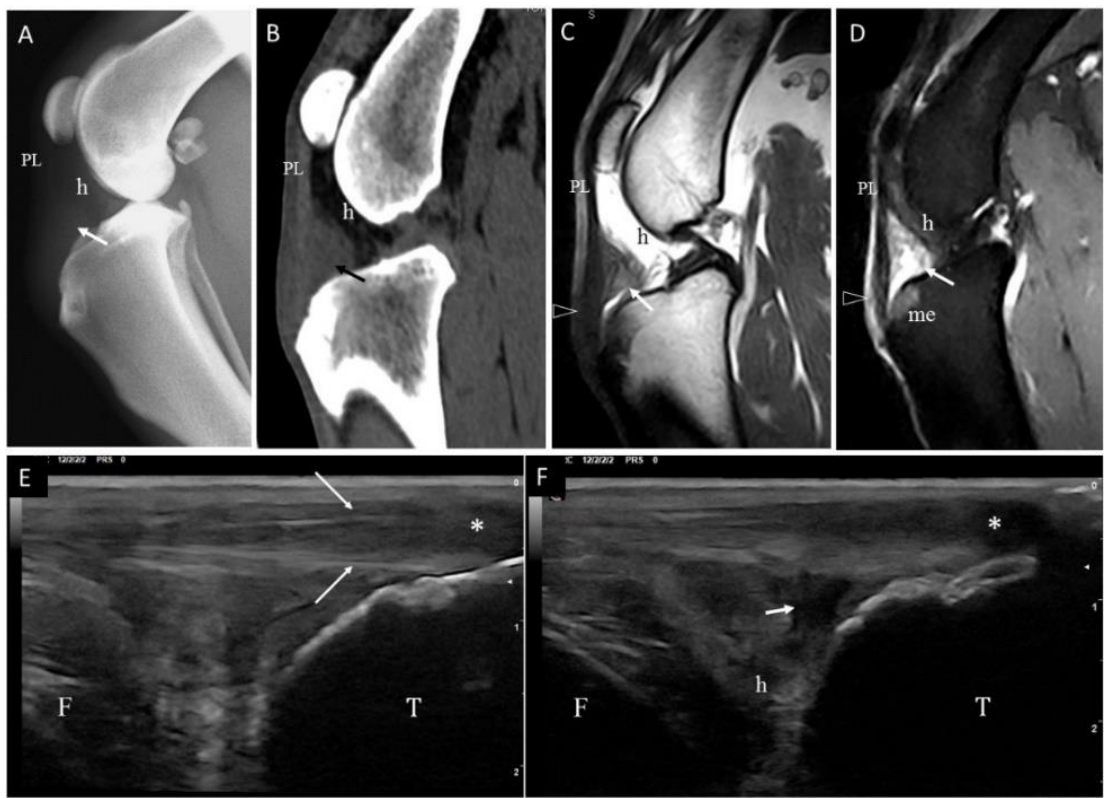

Fig. 1. Comparative diagnostic imaging of the right stifle joint. (A): Mediolateral radiograph. (B): Midsagittal multiplanar reformatted CT image. (C): Midsagittal T2-weighted and (D): gradient-echo STIR MRI images. (E, F): Longitudinal sonograms of the cranial aspect of the stifle joint. On radiographic image only the proximal part of the patellar ligament (PL) is visible with distinct margins while its distal part is ill-defined and surrounded by periligamentous soft tissue opacity (short arrow). The infrapatellar fat pad (h) shows loss of triangular shape. On CT image, the distal half of the patellar ligament is ill-defined ad surrounded by periligamentous accumulation of irregularly marginated material isoattenuating to the soft tissue (short arrow). The infrapatellar fat pad is caudally displaced. There are no signs of bone fractures or detached bone fragments at the tibial osteoligamentous junction. On MRI images, there is evident enlargement of the distal part of the PL (arrowheads) with intraligamentous signal inhomogeneity. The damaged part of the PL is surrounded by pathologic material, isointense on T1 and T2 weighted and hyperintense on STIR sequences, resembling a clot (short arrow). The pathologic material displaces the cranial aspect of the infrapatellar fat pad (h). A focal area of bone hyperintensity suggestive of bone marrow edema-like lesion (me) is evident on STIR image at level of the cranial aspect of tibial plateau, close to the osteoligamentous junction. On US the PL shows enlargement of its distal part and heterogeneous echotexture with intraligamentous anechoic defect (asterisks). The paratenon (long arrows) appears unaffected. The infrapatellar fat pad (h) appears inhomogeneous, hypoechoic with a small inner fluid collection. T: Tibia; F: Femur.
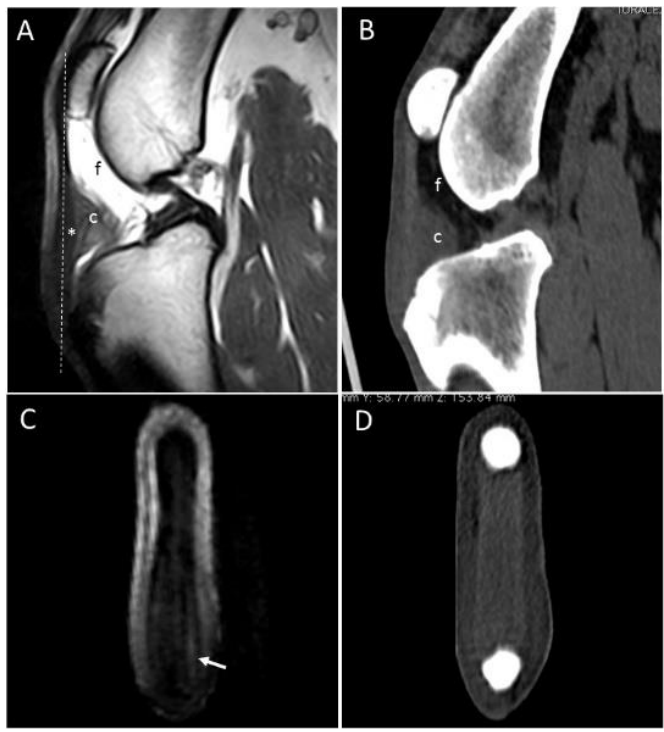

Fig. 2. Midagittal oblique multiplanar reformatted bSSFP MRI (A) and CT (B) images of the patellar ligament (PL). Dorsal oblique multiplanar reformatted bSSFP MRI (C) and CT (D) images obtained orienting slice plane in the centre of the ligament (dotted line in image A). There is thickening of the distal half of PL (A - asterisk) and pathologic material (c), resembling a clot, adjacent to the inner surface of PL and displacing the infrapatellar fat pad (f). A small linear defect is clearly visible on MRI images (C-arrow) in disto-medial position within PL structure. Such detail is not detectable on CT image obtained at the same level (D). 

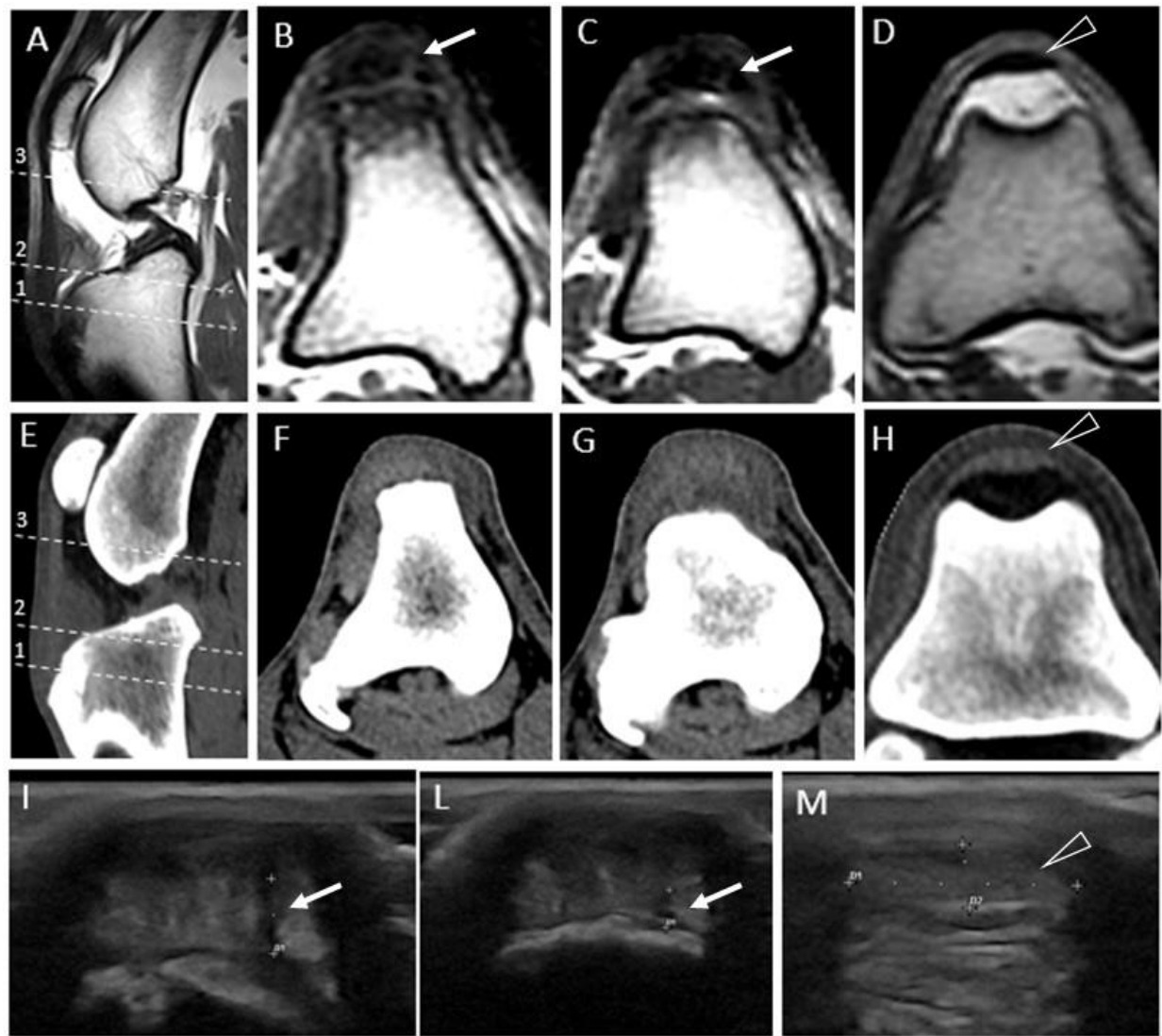

Fig. 3. (A): Midagittal multiplanar reformatted bSSFP MRI image of the patellar ligament (PL). (B,C,D): Transverse multiplanar reformatted bSSFP MRI images at different levels of PL course. Image B is obtained orienting the oblique transverse plane at level 1 of image A. Image $\mathrm{C}$ is obtained orienting the oblique transverse plane at level 2 of image A. Image D is obtained orienting the oblique transverse plane at level 3 of image A. (E): Midsagittal multiplanar reformatted CT image of the patellar ligament (PL). $(\mathbf{F}, \mathbf{G}, \mathbf{H})$ : Transverse multiplanar CT images at different levels of PL course. Image F is obtained orienting the oblique transverse plane at level 1 of image A. Image $\mathrm{G}$ is obtained orienting the oblique transverse plane at level 2 of image A. Image $\mathrm{H}$ is obtained orienting the oblique transverse plane at level 3 of image A. (I): Transverse sonographic image of the PL at level 1 of image A. (L): Transverse sonographic image of the PL at level 2 of image A. (M): Transverse sonographic image of the PL at level 3 of image A. On midsagittal MRI and CT images thickening of the distal PL half is evident. Transverse MRI images at different levels show in detail the caudo-cranial extension of the tear within PL sections: at most distal part of the PL (B - level 1 of Fig. A) the defect affects a large portion of ligament section while it became smaller proximally being confined to the most caudal part of PL section (C- level 2 of Fig. A). Images D and H shows normal PL sections (arrowhead) with homogeneous signal and attenuation respectively (level 3 of Fig. A). Such details are not evident on CT images (F, G) but easily assessable on correspondent transverse sonographic images as hypoechoic linear defects (I,L-arrows).

Similarly, in human radiology if patella alta is detected on a knee radiograph in a trauma setting, further imaging is preferred in order to rule out or characterise eventual patellar tendon rupture (Bartalena et al., 2010). Contrary to plain radiographs, CT permits tomographic imaging through the patellofemoral joint without structure overlap. When evaluated for the assessment of the normal canine stifle, CT scans provided excellent images of the joint anatomy with good diagnostic detail of tendons and ligament texture, albeit less clearly defined than MR images (Soler et al., 2007). In the human medical literature, a focal decrease in attenuation and tendon thickening are considered specific CT findings indicative of partial rupture. Very small partial tears may be missed, however, due to limited contrast and spatial resolution for soft tissue (Kälebo et al., 1990). Unfortunately, the diagnostic sensitivity of CT for partial PL injuries in dogs remains as yet undescribed in the veterinary medical literature. In our case, although we observed a higher contrast resolution on soft tissue articular components in CT images compared to radiographs due to the volumetric near-isotropic acquisition, no additional information regarding PL integrity was obtained. The damaged PL area appeared thickened but homogenously isoattenuating to periarticular soft tissue, without any evidence of structural discontinuity or attenuation changes. This finding is in accordance with the human medical literature, which describes CT scans as useful tools for diagnosing tendon avulsion but not for the 
detection of mid-substance lesions (Rosso et al., 2015). On the other hand, the CT images, endowed with high contrast resolution for mineralised tissue, clarified the cortical bone integrity at the level of the tibial tuberosity, overcoming the limit of radiographic superimposition. In our case the major contribution of CT was in the assessment of the osteoligamentous junction and the exclusion of distal PL avulsion.

In MRI images, normal ligamentous structures appear as strongly and homogeneously hypointense bands (Soler et al., 2007; Hodgson et al., 2012) in all sequences, and thus any intraligamentous lesions are easily detectable as an area of bright signal. In accordance with reports regarding traumatic injuries in humans (Hodgson et al., 2012), the main MRI findings of the present study included the following: PL thickening; a large periligamentous swelling that was hyperintense in fat-suppressing images, suggestive of paratenonitis and periligamentous hematoma (Hodgson et al., 2012); and a hyperintense linear signal abnormality crossing part of the ligament, suggestive of fissuration.

As previously described, bSSFP appeared the most useful sequence for PL assessment, since its volumetric dataset could be multiplanarly reformatted and a pure dorsal image passing through the ligament centre could be obtained (Ricciardi, 2018). Whereas this precise dorsal-oriented reconstruction clearly showed the presumed fissuration throughout its ventro-dorsal extension (Fig. 2-A,C), other non-volumetric sequences demonstrated only part of the lesion in transverse images, depending on the slice alignment. Endowed with high soft-tissue contrast resolution, MRI also permitted a broad overview of all articular components, with easy individuation of the PL injury. Furthermore, other ligament injuries, in particular those affecting cruciate ligaments, could be easily excluded. The normal PL is very readily assessable via US, according to both the human and veterinary medical literature, and is described as a striated hyper-reflective structure hyperechoic to the patellar fat pad. Collagen bundles reflect sound waves to the transducer, giving an echogenic fibrillary pattern to the normal PL in both short and long axes. The paratenon appears as a hyperreflective solid line around the PL tissue (Soler et al., 2007; Chang and Miller, 2009; McNally, 2014). A tear may be seen as a hypoechoic area interrupting ligament fibres and extending across the ligament as a fullthickness complete or partial tear (Hodgson et al., 2012).

This pattern of intraligamentous lesion was confirmed in our US images, which allowed an in-depth-view of the PL structure at high spatial resolution and subsequent high detail of fibrillary pattern. The identification of the lesion and the evaluation of its extension were clearly assessed due to the high contrast resolution between the hypoechogenicity of the fibrillary gap and the echogenicity of the normal ligament tissue (Fig. 3-I,L).

The US images also allowed a presumptive imaging diagnosis of partial distal PL fissuration demonstrating: 1) the longitudinal direction of the lesion, limited to the distal half of the PL, 2) integrity of paratenon and 3) partial caudo-cranial extension in transverse images obtained in proximity to the ligament centre. In human ultrasonography, similar focal disruptions of the fibrillary pattern in US images are considered specific to partial tears of the PL (Hodgson et al., 2012).

In human medicine, circumferential hypoechogenicity around the tendon is suggestive of paratenonitis (Hodgson et al., 2012). In our patient, the pathologic material interposed between PL and infrapatellar fat pad appeared nonspecific and, in the different images, its pattern was suggestive of a blood clot and soft tissue inflammation.

Based on a comparison of the four imaging techniques, common imaging findings included PL thickening and periligamentous swelling compatible with hematoma or soft tissue inflammation. However, in the authors' opinion, these findings were of minor diagnostic importance with respect to the visualisation of the PL tear, which should be the goal of any imaging assessment in order to orientate subsequent therapeutic treatment.

The intraligamentous lesion was seen clearly only in the MRI and US images. Furthermore, the US images also provided superior visualisation of the fibrillary PL architecture and further detail regarding the position, extension and paratenon involvement of the fibrillary gap (i.e. tendon fibre discontinuity). In this case, the US images confirmed and clarified tear extension within the structure of the PL, with respect to both the cranial margin of the ligament (not involved in the fissure) and the paratenon (also unaffected by the lesion). Such details were not clearly assessable in the MRI images due to the latter's inferior spatial resolution. This observation suggests that US may offer slightly superior detail compared to MRI for the accurate imaging diagnosis of partial PL tears.

Another key advantage of ultrasound compared to MRI is the ability to dynamically assess tendons and ligaments, allowing visualisation both from different angles and under stress (Hodgson et al., 2012). However, in our case the tear extent did not vary during knee joint flexion-extension, probably due to its longitudinal path within the PL.

Besides damaging the PL, traumatic force also exerted an effect on the tibial tuberosity. At this level, the CT images excluded any sign of microfracture or avulsion, but failed to show significant bone attenuation changes. In contrast, MRI appeared to be the most sensitive technique regarding the assessment of bone damage, 
showing a focal area of increased signal in STIR sequences at the distal osteoligamentous junction (Fig. 1-D).

Such intraosseous signal changes, deriving from haemorrhage, hyperemia, edema and microfractures, have been described as bone marrow edema-like lesions (BMELLs) and may be found as a consequence of direct bone bruises or from traction forces occurring during an avulsion injury (Eriksen and Ringe, 2012). Regardless of the origin of this bone lesion (direct contusive vs tensile forces), its detection in an imaging study should be considered noteworthy, since it not only provided an insight into the actual extent of knee damage, but also since BMELLs are a considerable pain generator, as described in the human medical literature (Starr et al., 2008).

As also reported in human radiology, the present findings confirmed MRI and US as the most valuable imaging tools for the assessment of a PL partial tear, demonstrating high accuracy in the detection of ligamentous damage. Whereas MRI can provide global joint assessment at high detail for intraligamentous and intraosseous signal changes, sonography should above all be considered a focused examination, concentrating on the area of PL abnormality in order to obtain a deeper characterisation of fibrillary damage and paratenon integrity. Radiographic and CT examination proved less useful for PL injury characterisation. In the authors' opinion, their utility is limited to the early individuation of damaged compartments and avulsion injury exclusion.

In conclusion, our preliminary findings justify the combined use of MRI and US as accurate imaging techniques for PL injuries after clinical examination and survey radiographs. Further prospective studies are needed in order to evaluate the sensitivity and specificity of such imaging techniques, using large cohorts of dogs, for different types of PL injury.

\section{Acknowledgments}

The authors wish to thank Dr. Giuseppe Calamo, DVM in Ostuni (BR), Italy and the staff of the Pingry Veterinary Hospital of Bari, Italy for their assistance with data collection.

\section{Conflict of interest}

The authors declare that there is no conflict of interests.

\section{References}

Aron, D.N., Selcer, B.A. and Smith, J.D. 1997. Autogenous tensor fascia lata graft replacement of the patellar ligament in a dog. Vet. Comp. Orthop. Traumatol. 10, 141-145.

Barone, R. 2003. Anatomia comparata dei mammiferi domestici: osteologia, Vol. 2, $3^{\text {rd }}$ ed. Edagricole, Bologna, pp: 269.

Bartalena, T., Rinaldi, M.F., De Luca, C. and Rimondi, E. 2010. Patellar Tendon Rupture: Radiologic and
Ultrasonographic Findings. West J. Emerg. Med. 11, 90-91.

Bloomberg, M.S. and Parker, R.B. 1984. Chronic lameness in the dog due to delayed diagnosis of disruption of patellar ligament. J. Am. Anim. Hosp. Assoc. 20, 899-904.

Chang, A. and Miller, T.T. 2009. Imaging of tendons. Sports Health 1, 293-300.

Chiang, Y.P., Wang, T.G. and Shiau-Fu Hsieh, S.F. 2013. Application of Ultrasound in Sports Injury. J. Med. Ultrasound 21, 1-8.

Culvenor, J.A. 1988. Fascia lata flap to reinforce repair of patellar ligament injuries in the dog and cat. J. Small Anim. Pract. 29, 559-563.

Das, S., Thorne, R., Lorenz N.D., Clarke, S.P., Madden, M., Langley-Hobbs, S.J., Perry, K.L., Burton, N.J., Moores, A.L. and Mosley, J.R. 2014. Patellar ligament rupture in the dog: repair methods and patient outcomes in 43 cases. Vet. Rec. 175, 370.

Eriksen, E.F. and Ringe, J.D. 2012. Bone marrow lesions: a universal bone response to injury? Rheumatol. Int. 32, 575-584.

Gemmill, T.J. and Carmichael, S. 2003. Complete patellar ligament replacement using a fascia lata autograft in a dog. J. Small Anim. Pract. 44(10), 456-459.

Greis, P.F., Holmstrom, M.C. and Lahav, A. 2005. Surgical treatment options for patella tendon rupture, Part I: Acute. Orthopedics. 28, 672-679.

Hall, B.K. 2007. Fins Into Limbs: Evolution, Development, and Transformation. University of Chicago Press, Chicago, IL USA, pp: 328.

Hodgson, R.J., O'Connor, P.J. and Grainger, A.J. 2012. Tendon and ligament imaging. Br. J. Radiol. 85, 1157-1172.

Insall, J. and Salvati, E. 1971. Patellar position in the normal knee joint. Radiology 101, 101-104.

Kälebo, P., Goksör, L.A. and Swärd, L. 1990. Peterson Soft-Tissue Radiography, Computed Tomography, and Ultrasonography of Partial Achilles Tendon Ruptures. Acta Radiol. 31, 565-570.

Lin, T.W., Cardenas, L. and Soslowsky, L.J. 2004. Biomechanics of tendon injury and repair. J. Biomech. 37, 865-877.

McNally, E. 2014. Practical musculoskeletal ultrasound. $2^{\text {nd }}$ edition. Elsevier Churchill Livingstone, Oxford, pp: 229.

Melloni, P., Marin, A., Veintemillas M.T., Valls, R., Sabadell, E.S. and Sant Quirze Del Valles, E.S. 2012. Magnetic resonance imaging in patellar tendon abnormalities: A pictorial review - ESSR meeting, Innsbruck, June 28-30.

Mostafa, A.A. 2008. Musculoskeletal conformation of the normal and diseased canine stifles with emphasis on patellar luxation and cranial cruciate 
ligament deficiency. PhD Thesis, Department of Veterinary Clinical Science, University of Illinois at Urbana-Champaign (UIUC), USA, pp: 27.

Ricciardi, M. 2018. Principles and applications of the balanced steady-state free precession sequence in small animal low-field MRI. Vet. Res. Commun. 42, 65-86.

Rosso, F., Bonasia, D.E., Cottino, U., Dettoni, F., Bruzzone, M. and Rossi, R. 2015. Patellar tendon: From tendinopathy to rupture. Asia Pac. J. Sports Med. Arthrosc. Rehabil. Technol. 2, 99-107.

Schenck Jr, R.C. 2001. Injuries of the knee. In: Heckman JD, Bucholz RW, editors. Rockwood and Green's fractures in adults. $5^{\text {th }}$ ed. Lippincott Williams \& Wilkins, Philadelphia, pp: 1843-1938.

Soler, M., Murciano, J., Latorre, R., Belda, E.,
Rodríguez, M.J. and Agut, A. 2007. Ultrasonographic, computed tomographic and magnetic resonance imaging anatomy of the normal canine stifle joint. Vet. J. 174, 351-361.

Starr, A.M., Wessely, M.A., Albastaki, U., PierreJerome, C. and Kettner, N.W. 2008. Bone marrow edema: pathophysiology, differential diagnosis, and imaging. Acta Radiol. 49, 771-786.

Sumner-Smith, G. 1993. Gait analysis and orthopedic examination. In: Slatter D (ed.): Textbook of small animal surgery, Vol. 2. $2^{\text {nd }}$ ed. Saunders, Philadelphia, pp: 1577.

Torriani, M. and Kattapuram, S.V. 2003. Musculoskeletal ultrasound: an alternative imaging modality for sports-related injuries. Top. Magn. Reson. Imaging 14, 103-111. 